\title{
Ochratoxin $A$ is not detectable in renal and testicular tumours
}

\author{
Nader Fahmy, MD, FRCSC; ${ }^{*}$ Mark Woo,; Mona Alameldin, MD; ${ }^{+}$Kyle MacDonald, MBSc; ${ }^{\text {;s }}$ \\ Lee W. Goneau, BSc,s Peter Cadieux, PhD; Stephen E. Pautler, MD, FRCSC ${ }^{*}$
}

*Division of Urology, Department of Surgery, Schulich School of Medicine \& Dentistry, Western University, London, ON, 'Department of Pathology, Schulich School of Medicine \& Dentistry, Western University, London, ON; §Department of Microbiology \& Immunology, Schulich School of Medicine \& Dentistry, Western University, London, ON; *Division of Surgical Oncology, Department of Oncology, Schulich School of Medicine \& Dentistry, Western University, London, ON

Cite as: Can Urol Assoc J 2014;8(1-2):40-6. http://dx.doi.org/10.5489/cuaj.1240 Published online February 10, 2014.

\section{Abstract}

Introduction: Ochratoxin-A (OTA) is one of the most abundant food-contaminating mycotoxins, known for its nephrotoxicity, neurotoxicity, gonadotoxicity, teratogenicity, immunosuppression and carcinogenesis. OTA has been linked to several genitourinary pathologies, including Balkan nephropathy and genitourinary malignancies. We examine OTA levels in serum samples and tumour specimens collected from patients with renal and testicular tumours.

Methods: Frozen samples were obtained from the Ontario Tumour Bank. Serum specimens, along with renal and testicular tumour biopsies, were included in this study. Normal tissue from the negative surgical margins of each tumour served as a control. OTA levels in serum was measured using the enzyme-linked immunosorbent assay (ELISA), while OTA detection in tissue specimens was determined using immunohistochemistry (IHC).

Results: We included specimens collected from 56 patients (36 men and 20 women). Histopathology of the 52 renal tumours included 31 (60\%) conventional type renal cell carcinomas (RCC), 5 (10\%) chromophobe RCC, 5 (10\%) papillary RCC, 1 (2\%) oncocytoma and 10 (19\%) upper tract urothelial carcinoma (UC). The 4 testicular tumours included 1 seminomatous (25\%) germ cell tumour and 3 (75\%) non-seminomatous germ cell tumours. OTA was detected in the serum of renal tumour patients, with a range from 0.004 to $0.25 \mathrm{ng} / \mathrm{mL}$ (mean: 0.07 and median $0.06 \mathrm{ng} / \mathrm{mL}$ ). There was no OTA signal detected by IHC staining in all tested renal and testicular tumours.

Conclusions: The OTA levels detected in the serum of patients were highly variable and relatively low. No OTA was detected in the tissue samples.

\section{Introduction}

Ochratoxins (A, B, and C) are a group of mycotoxins produced by several species of Aspergillus and Penicillium.
Out of more than 300 currently described mycotoxins, ochratoxin A (OTA) is one of the most toxic and abundant. It contaminates a variety of animal feeds and human food and beverages when stored in hot and moist environments. ${ }^{1}$ In most countries, the main sources for OTA human food contamination are cereal grains followed by grape products and wine ${ }^{2}$ and to a lesser extent coffee, beans, cocoa, beer, nuts, coriander, black pepper, chili powder and ginger. ${ }^{3}$

Animal studies have shown that OTA is mostly absorbed by the small intestine. In the blood, it mainly binds to albumin. Because of its small molecular weight, OTA is readily filtered by the glomerular membrane and accumulates in kidneys. ${ }^{3}$ OTA was detected in human milk ${ }^{3}$ and can freely cross the blood placental barrier and was thus associated with DNA adducts in fetal kidneys and testicular tissues. ${ }^{4}$

OTA is nephrotoxic, ${ }^{5}$ teratogenic, ${ }^{6}$ immunosuppressive $^{7}$ and carcinogenic ${ }^{8}$ in many animal species, including rodents, poultry, canine, fish and swine. Oral administration of OTA caused renal tumours in mice ${ }^{9}$ and rats. ${ }^{8}$ Human studies have linked endemic Balkan nephropathy (EBN) to areas with high OTA food contamination. ${ }^{3}$ Furthermore, the consumption of OTA-contaminated pork and coffee has been correlated to testicular cancer incidence in 20 countries..$^{10}$ Therefore, we propose a theoretical causal role for OTA in inducing urothelial, renal and testicular cancers.

The mechanism by which OTA could induce cancer remains unclear. However, to date, there have been no satisfactory records of a direct association between OTA exposure and human cancer; therefore, the International Agency for Research on Cancer (IARC) has classified OTA as a possible human carcinogen (Group 2B). ${ }^{11}$ Ever since this classification, growing worldwide attention has focused on estimating OTA content in diet and limiting human exposure. We examine human OTA levels in serum from patients with documented renal and urothelial tumours and determine its concentration in renal, urothelial and testicular tumours and adjacent normal tissues. 


\section{Methods}

\section{Material}

This research was approved by our institutional Research Ethics Board. Frozen samples were obtained from the Ontario Tumour Bank, a program established by the Ontario Institute for Cancer Research (OICR). It is a province-wide biorepository and data bank focused on collecting tumour-related human biospecimens. Specimens included frozen serum and fresh-frozen tissue biopsies collected from patients with renal or testicular tumours. Each case in the OICR tumour bank has well-characterized clinical and pathological information. All frozen tissue biopsies included tumour specimens and matching controls collected from their corresponding non-neoplastic negative surgical margins. All samples were stored at $-80^{\circ} \mathrm{C}$.

\section{Measurements of serum OTA concentration}

The level of OTA in the serum was tested following the manufacturer instructions using the commercially available Quantitative Assay for OTA in Human and Animal Serum and Milk Enzyme Linked Immunosorbent Assay (ELISA) 96-well kit (Helica Biosystems, Inc., Santa Ana, CA). Supplied vials with known serial OTA dilutions in $70 \%$ methanol served as a calibration standard. All samples were run in duplicates. The optical densities (OD) of the assay solutions were then measured using a spectrophotometer with a 450-nm filtre. A dose-response curve of OD against OTA concentration was constructed from the standards using GraphPad Prism version 4 (GraphPad Software, Inc., La Jolla, CA). A standard equation was established with curve estimation. Serum sample concentrations were then calculated $(\mathrm{ng} / \mathrm{mL})$ by interpolation from the equation using their measured optical densities. The mean of each duplicate sample was computed and reported.

\section{Tissue preparation}

Collected frozen tissues were cut into $4-\mu \mathrm{m}$ sections using the Leica CM3050 S Cryostat (Leica Biosystems GmbH, Nussloch, Germany). Pairs of contiguous tissue sections were mounted on VWR Superfrost glass microslides (VWR international LCC, Radnor, PA) and stored at $-80^{\circ} \mathrm{C}$.

\section{Immunohistochemistry}

\section{Testing OTA expression in frozen tissues}

Immunohistochemistry (IHC) was performed on the frozen tumour and their matching non-neoplastic negative surgical margin to determine the presence and location of OTA. Following defrosting of the slides, the tissue was fixed for 10 seconds in cold acetone. A 10-second treatment with $3 \%$ hydrogen peroxide in methanol was then performed to block endogenous peroxidase activity. Blocking was accomplished using 20\% heat-inactivated normal horse serum (Vector Laboratories, Inc., Burlingame, CA) in phosphate buffered saline (PBS) for 20 minutes. The samples were then incubated overnight at $4^{\circ} \mathrm{C}$ with a rabbit polyclonal antibody raised against OTA- keyhole limpet hemocyanin (KLH) conjugates (MyBioSource, Inc., San Diego, CA) at a $1: 50$ dilution in $20 \%$ normal horse serum. A subsequent 30-minute incubation was performed at room temperature with Peroxidase-AffiniPure Goat Anti-Rabbit HRP-polymer IgG antibody (Bethyl Laboratories Inc., Montgomery, TX ) at a dilution of $1: 500$ in a solution of $2.5 \%$ skimmed milk and $5 \%$ horse serum (for additional blocking) in PBS. A 3, 3'-diaminobenzidine (DAB Peroxidase Substrate Kit, Vector Laboratories, Inc., Burlingame, CA) was used for visualization. Sections were counterstained with hematoxilyn and dehydrated using the Leica Autostainer XL Staining System ST5010 (Leica Biosystems GmbH, Nussloch, Germany). Slides were mounted in Surgipath Micromount mounting medium (Leica Biosystems Richmond Inc, Richmond, IL). Samples were examined by one pathologist using an Olympus BX51 optical microscope (Olympus America Inc, Center Valley, PA). Pictures were captured using a high quality microscope SPOT Insight $2 \mathrm{Mb}$ colour camera and analyzed using SPOT Basic Software version 4.1 (SPOT Imaging Solutions, Sterling Heights, MI).

\section{Positive and negative control testing}

An Amersham Hybond ECL Nitrocellulose Membrane (GE Healthcare UK Limited, Buckinghamshire, UK) was initially secured in a miniblot apparatus (Schleicher and Scheull, Inc., Keene, NH). After connecting the miniblot apparatus to low wall suction, duplicates of $400 \mu \mathrm{L}$ of each of the following was added to separate wells:

- Negative controls: PBS and Serial dilution of Fetal Bovine Serum (FBS) (Sigma-Aldrich Corporation, St. Louis, MI) in PBS to rule out potential cross reactivity.

- Positive controls: Serial dilutions of standard OTA (Helica Biosystems, Inc., Santa Ana, CA).

A $200-\mu \mathrm{L}$ of serially diluted OTA was also tested in different wells (Fig. 1).

Previously described IHC technique was applied to the membrane. Following the addition of the DBA substrate, the membrane was rinsed in distilled water and allowed to air dry for 1 hour. The membrane was then scanned using a personal high resolution Lexmark 5200 Series photo scanner (Lexmark International, Inc., Lexington, KY). 
Fahmy et al.

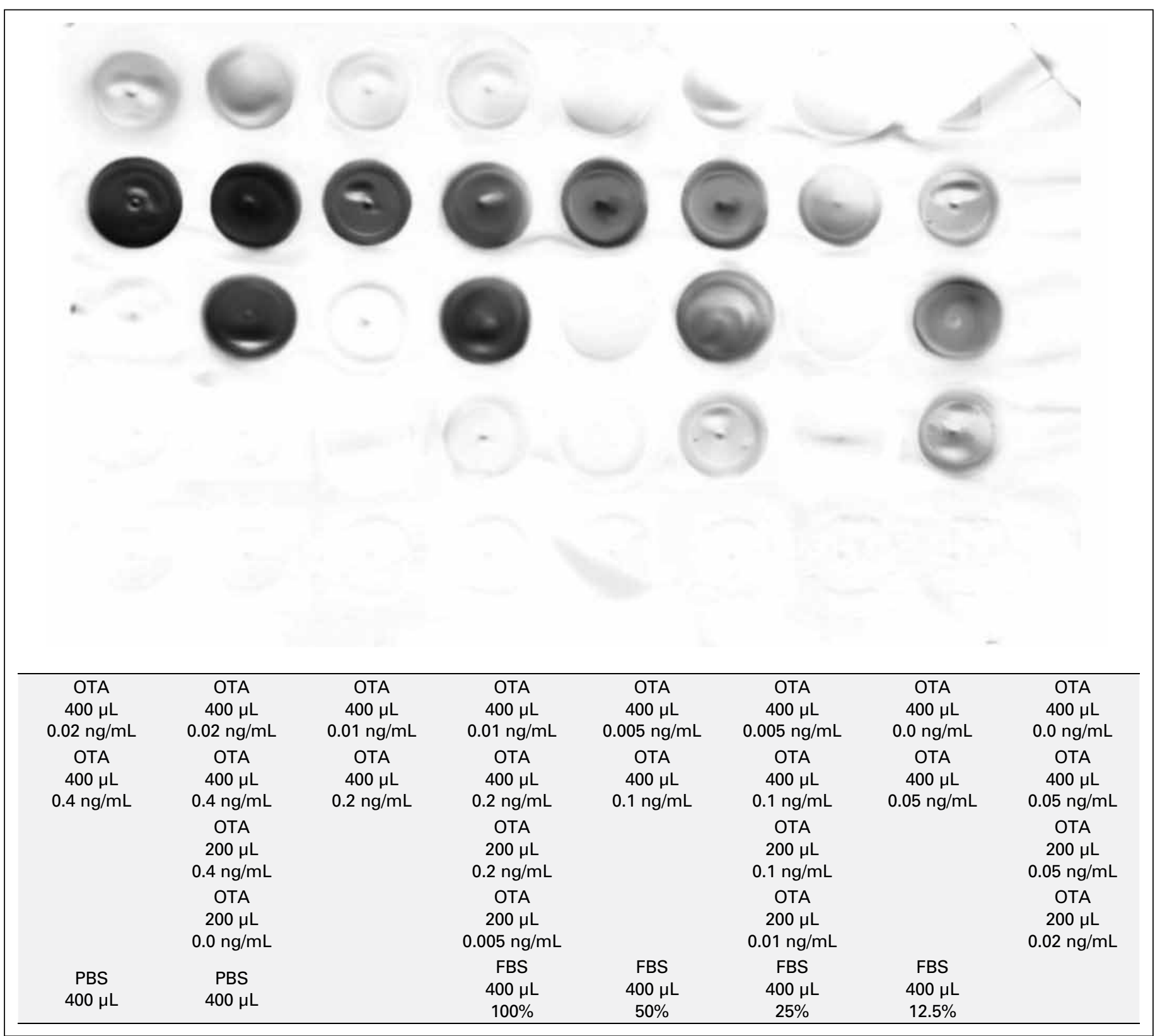

Fig. 1. Immunohistochemistry staining of an Ochratoxin-A coated nitrocellulose membrane.

\section{Statistical analysis}

Data were assessed for normality distribution. A non-parametric Kruskal-Wallis test was used to evaluate serum OTA concentration among different histopathological tumours. Differences between mean gender serum OTA levels were compared using a Student t-test for unpaired data. Results were considered significant for $p$ values $<0.05$. Statistical analyses were performed using an IBM SPSS software version 21 (IBM, New York, NY).

\section{Results}

A total of 56 tumour tissue specimens (52 renal, 4 testicular), along with their corresponding non-neoplastic negative surgical tissue margins, were provided by the Ontario tumour bank. These frozen specimens were collected from 56 patients ( 36 men and 20 women). We also included 37 serum samples preoperatively collected from renal tumour patients. Patient age ranged from 37 to 82 years, with a mean of 62.8 years (median: 68 years). 


\section{Tumour characteristics}

Of the 52 renal tumours, frozen renal tissue samples were collected from patients who had undergone 5 laparoscopic radical nephrectomies, 37 open radical nephrectomies and 10 nephroureterectomies. The mean tumour size was $6.52 \mathrm{~cm}$ (median $5.5 \mathrm{~cm}$ ) and ranged from 2.8 to $14 \mathrm{~cm}$. Three patients died of their disease. The histopathology of the included tumours included $31(60 \%)$ conventional type renal cell carcinoma (RCC), 5 (10\%) chromophobe, 5 (10\%) papillary, 1 (1\%) oncocytoma and 10 (19\%) upper tract urothelial carcinoma (UC) (Table 1).

Of the 4 frozen tissue samples from 4 inguinal orchiectomies, 1 patient had seminomatous germ cell tumour and 3 had non-seminomatous germ cell pathology. No accompanying frozen plasma samples were provided. Tumour size ranged from 2.5 to $4 \mathrm{~cm}$. The mean tumour size was $3.15 \mathrm{~cm}$ (Table 1).

\section{Levels of OTA in frozen plasma samples}

Frozen plasma samples were taken from 21 conventional RCC, 5 chromophobe RCC, 2 papillary RCC, 8 renal pelvis $\mathrm{UC}$ and 1 oncocytoma. We performed a quantitative determination of OTA in 37 frozen plasma samples collected from renal tumour patients. The detected serum OTA concentration ranged from 0.004 to $0.25 \mathrm{ng} / \mathrm{mL}$ (mean: 0.07 , median $0.06 \mathrm{ng} / \mathrm{mL}$ ) (Fig. 2). There was a tendency towards increased OTA levels in frozen plasma samples taken from patients who were treated for a conventional RCC or upper tract UC $(p<0.08)$.

\section{OTA localization in frozen tissue samples}

The OTA coated positive control portions of the nitrocellulose membrane were successfully stained by IHC. DAB substrate reacted with different OTA concentrations and

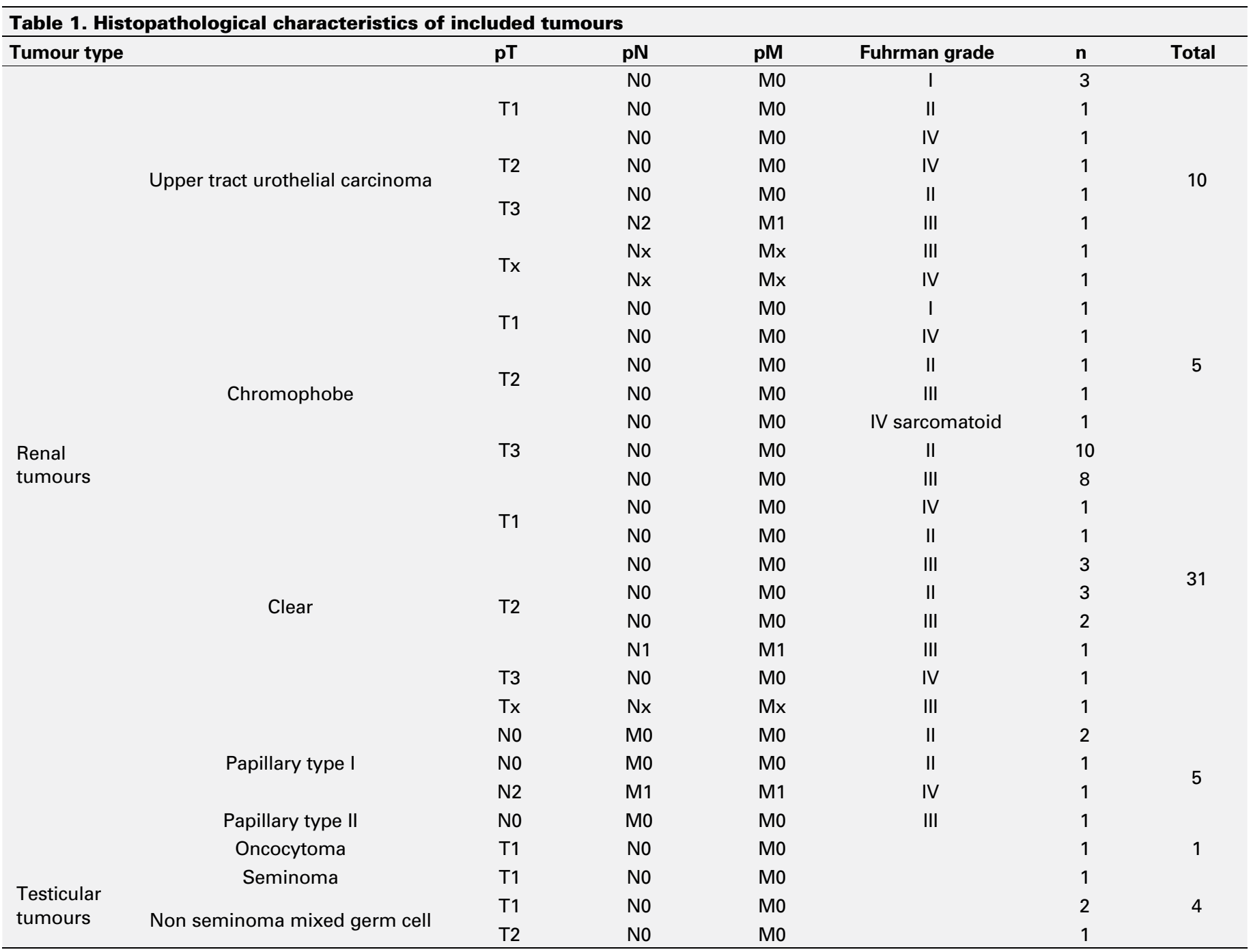


Fahmy et al.

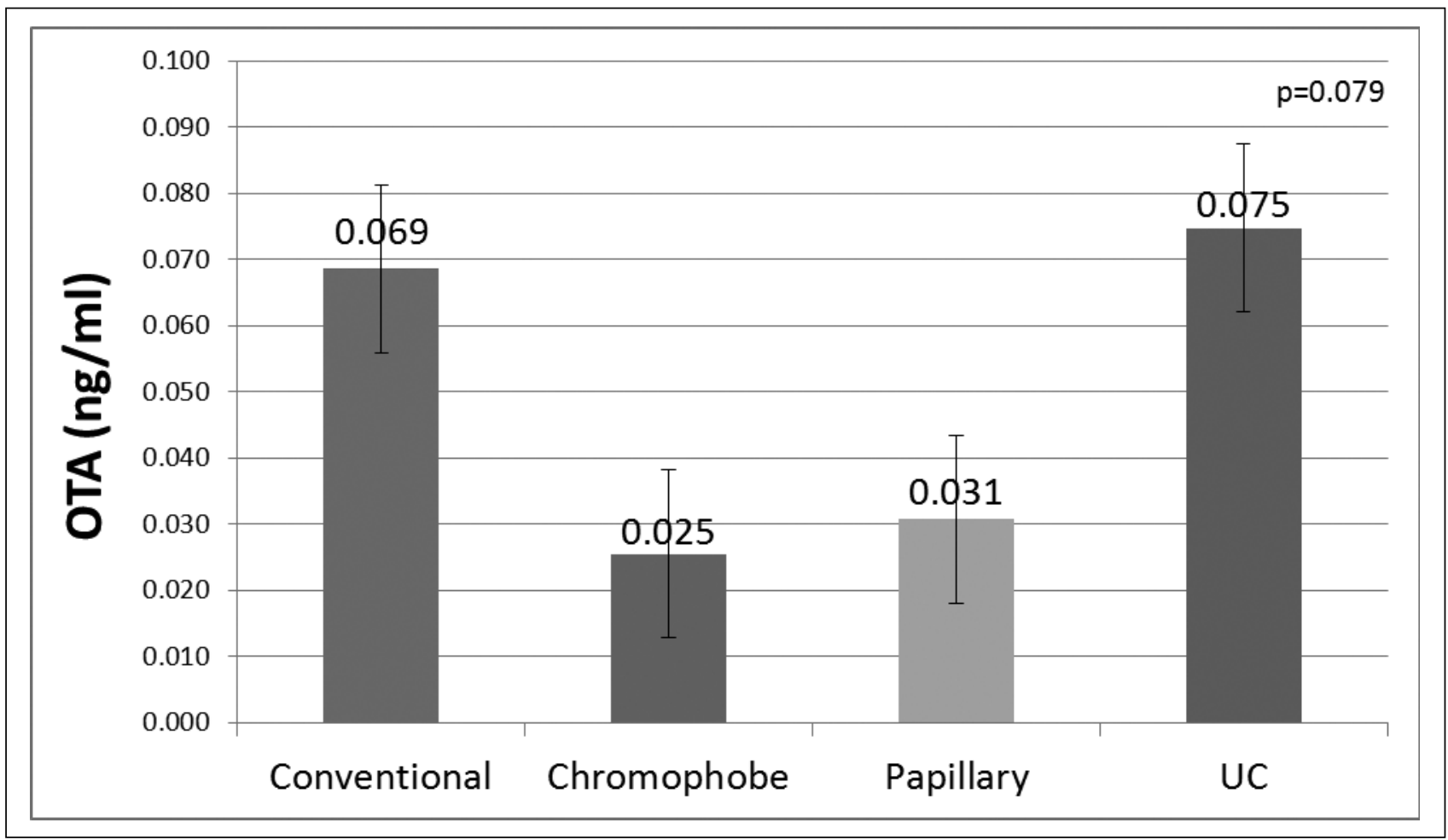

Fig. 2. Ochratoxin-A concentration in frozen serum tested by enzyme-linked immunosorbent assay (ELISA).

a spectrum of lighter colour gradients that proportionately correlated with serial OTA dilutions was obtained. The PBS negative control coated portions of the membrane did not show any staining and similarly there was no evidence of cross-reactivity with FBS. There was no OTA staining detected within all tested renal and testicular tissue samples.

\section{Discussion}

Evidence suggesting an OTA kidney cancer risk first arose when a significantly increased incidence of benign and malignant renal tumours was observed in OTA fed male rodents. ${ }^{3,4,9,12}$ This increase proportionately correlated with study duration ${ }^{12}$ and dose of OTA intake. ${ }^{4,9}$ Human studies have also indicated a higher incidence of EBN in areas where high human OTA food contamination exists. ${ }^{3}$ Renal functional and structural changes detected in EBN were similar to porcine induced OTA toxicity. ${ }^{13}$ In addition, when compared to controls with no urinary disorders, increased OTA serum levels were detected in UC and renal cancer patients living in Bulgaria ${ }^{14}$ and Egypt. ${ }^{15}$ Based on the previously described association of Balkan nephropathy to upper tract $U C,{ }^{16}$ OTA exposure may be associated with increased $\mathrm{UC}$ and renal cancer risks. A growing attention to OTA exposure led to further investigation of other potential associated tumour risks, including testicular cancers. An experimental study suggested that exposing fetal mice in utero to OTA was associated with an increased potential incidence of testicular and kidney DNA adducts. ${ }^{4}$ Furthermore, human consumption of OTA-contaminated pork and coffee was correlated to testicular cancer incidence in 20 countries. ${ }^{10}$

Based on all previous findings, a human renal and/or testicular cancer risk was linked to OTA exposure and a causal relationship has been suggested. To date, OTA cancer risk to exposed human populations is still unclear and there have been no properly conducted epidemiologic case controlled studies examining this risk. However, human OTA cancer risk has been indirectly investigated. OTA was either externally tested by measuring airborne OTA in inhaled air ${ }^{17}$ or contaminated human diet, ${ }^{18}$ and/or internally tested by investigating OTA levels in biological fluids and tissues. Epidemiological studies subsequently linked OTA existence to disease incidence. ${ }^{17}$ The aim of this work was to investigate OTA in human serum and tumour tissues taken from patients with renal, urothelial or testicular tumours.

Given its strong binding capacity to albumin, serum OTA levels are generally considered an adequate indicator for assessing OTA exposure. In this study, mean OTA concentration in frozen serum samples collected from 37 renal tumour patients was $0.07 \mathrm{ng} / \mathrm{mL}$ and ranged from 0.004 to $0.25 \mathrm{ng} / \mathrm{mL}$. Mean OTA levels tended to be higher in conventional RCC and upper tract UC. Despite the absence of normal control samples in this study, our patients with renal tumours had a considerably lower serum OTA concentrations than those previously reported in healthy Canadian individuals. Two previous Canadian studies reported mean serum OTA con- 
centrations of $0.88 \mathrm{ng} / \mathrm{mL}$ (range $0.29-2.37 \mathrm{ng} / \mathrm{mL}$ ) in $100 \%$ of the tested healthy individuals ${ }^{19}$ and $1.13 \mathrm{ng} / \mathrm{mL}$ (range $<0.50->20.0 \mathrm{ng} / \mathrm{mL}$ ) in $40 \%$ of both individuals with and without renal impairment. ${ }^{20}$ The former study was restricted by a methodology that could only detect OTA concentrations higher than $0.15 \mathrm{ng} / \mathrm{mL}$ and there was no detection limit specified by the latter study.

Other studies have also investigated serum OTA levels in a variety of urinary disorders with considerable variability. Most of these studies included European or North African populations. Many of these studies have collectively reported OTA serum levels in individuals living in areas with endemic renal disorders, although most have not reported OTA serum concentrations in renal tumour patients. Mean serum OTA in individuals with no history of urinary disorders ranged from 0.1 to $2 \mathrm{ng} / \mathrm{mL}^{3,21}$ A sample of patients from Turkey, ${ }^{22}$ Tunisia, ${ }^{23}$ Egypt, ${ }^{15}$ Bulgaria ${ }^{14}$ and Poland ${ }^{21}$ suffering from renal diseases and UC had significantly higher mean serum OTA levels than healthy controls. The highest reported OTA serum levels ranged from 35 to $100 \mathrm{ng} / \mathrm{mL}^{3}{ }^{3}$ In contrast, 2 studies from Pakistan ${ }^{24}$ and Germany ${ }^{25}$ did not detect any differences in OTA serum concentration among patients with or without urinary disorders. The mean reported blood OTA for both groups was $0.3 \mathrm{ng} / \mathrm{mL}$ in the former study and $0.4 \mathrm{ng} / \mathrm{mL}$ in the latter.

Internal measures for computing human exposure cannot recognize sources of contamination. In this study, data on OTA dietary exposure were not available. Nevertheless, estimating human exposure is extremely challenging and may be inaccurate due to the presence of various confounding factors. OTA concentrations in tested food may not project actual human consumption due to the variable effects of cooking and food processing on OTA. ${ }^{26}$ In addition, food OTA content varies greatly, not only within different geographical locations, ${ }^{26}$ but also within batches of crops taken from a same location. ${ }^{3}$

The current manuscript is the first to test OTA localization in actual human tumours. The absence of a readily available human tissue with documented OTA contamination to serve as a positive control for IHC staining presented a significant challenge. We failed to detect any OTA signal by IHC despite spiking tumour tissues with different concentrations of OTA standards. Based on previously published western blot and bacterial challenge techniques, ${ }^{27}$ we attempted IHC staining of an OTA-coated nitrocellulose membrane. Levels as low as $0.01 \mathrm{ng} / \mathrm{mL}$ of OTA were successfully detected, indicating that our IHC technique was suitable. There was, however, no evidence of OTA expression in all frozen human renal and testicular tissues tested in this study. To our knowledge, only one previous study attempted direct localization of OTA in human organs. ${ }^{21}$ OTA was examined in sections taken from the renal cortex of 19 patients with renal tumours who have undergone a nephrectomy in Poland. Using a reversed phase liquid chromatography with fluorescence detection, mean OTA level was $0.23 \mathrm{ng} / \mathrm{g}$ (median $0.25 \mathrm{ng} / \mathrm{g}$ ) and ranged from 0.15 to $0.45 \mathrm{ng} .{ }^{21}$ In the previous study, OTA concentration was measured in the renal cortex and not in the actual tumours. In addition, no OTA localization using tissue staining techniques was attempted. There was no correlation to the histological types of the tumours, and dietary OTA contamination was not reported.

In spite of the reported higher incidence of testicular cancer in patients consuming OTA-contaminated pork and coffee from 20 different countries, ${ }^{10}$ many other studies have contested OTA as a testicular carcinogen. These studies have failed to find any difference between the incidence of testicular cancer in OTA-exposed rodents and controls., ${ }^{9,28}$

Based on the findings of this study, we cannot support or refute any potential oncogenic risk for OTA. The absence of a detectable OTA signal in tissues and the lower OTA levels in blood in comparison to previous studies may have several interpretations. This may be a reflection of a different era with less OTA-food contamination due to greater awareness and stricter governmental controls or simply different food storing, processing and cooking techniques. A different detection methodology (IHC and ELISA rather than liquid chromatography) is another potential explanation. Since most animal studies were relatively short ( $<2$ years), the minimum required dose, frequency and duration of OTA exposure for tumour induction is unknown. The mechanism by which OTA may induce tumours and how long it takes for those tumours to be clinically detected is also still unclear. Not all OTA-fed animals developed renal tumours. It is still undetermined why most female and many male rodents appear to be immune to OTA tumour risks than others. OTA localization in the kidneys of mice given a single dose of $25 \mathrm{mg} / \mathrm{kg}$ and sacrificed within 48 hours was previously confirmed by IHC. OTA was detected in mice fed 15 minutes to 6 hours previous to sample collection and was highest at 3 hours. ${ }^{28}$ OTA half-life was only examined in 1 human volunteer and was reported to be 840 hours or 35.55 days. ${ }^{29}$ OTA may have induced some DNA adducts in exposed patients; this may have induced malignant tumours years later after the initial OTA has been cleared. The absence of OTA in tissues may also be explained by the fact that animal carcinogens might not necessarily have the same effects and pathogenesis on humans. A recent study has demonstrated that phosphorylared-S6 ribosomal protein, which is regulated by the mammalian target of rapamycin (mTOR), was consistently expressed in rat renal tumours in response to long-term exposure to dietary OTA ranging from 26 to 105 weeks. In contrast to the animal model, human RCC, upper urinary tract UC from cases of Balkan endemic nephropathy, and a human angiosarcoma were negative for p-S6. ${ }^{30}$ Lastly, this study may just be underpowered to detect any OTA in human tissues by IHC. 
Fahmy et al.

\section{Conclusion}

To our knowledge, this is the first study to test actual OTA in human renal and testicular tumours. It included a substantial number of patients and has validated a novel technique for OTA-positive control staining. Information from this study may serve as a foundation for designing bigger experimental and epidemiologic studies.

Acknowledgements: The source of funding for this project was a young investigator award from the Northeastern Section of the American Urological Association. Biological Materials were provided by the Ontario Tumour Bank, which is funded by the Ontario Institute for Cancer Research.

Competing interests: Dr. Fahmy, Mr. Woo, Dr. Alameldin, Mr. MacDonald, Mr. Goneau and Dr. Cadieux all declare no competing financial or personal interests. Dr. Pautler has received unrestricted grants-in-aid from Paladin and Sanofi. He is also participating in an ongoing clinical OAB trial with Astellas.

This paper has been peer-reviewed.

\section{References}

1. IARC. Ochratoxin A. IARC Monographs on the Evaluation of Carcinogenic Risk of Chemicals to Humans Overall Evaluations of Carcinogenicity: An Updating of IARC Monographs Volumes 7 to 42. Lyon, France: International Agency for Research on Cancer; 1987:271-2.

2. Visconti A, Perrone G, Cozzi G, et al. Managing ochratoxin A risk in the grape-wine food chain. Food Addit Contam Part A Chem Anal Control Expo Risk Assess 2008;25:193-202. http://dx.doi.org/10.1080/02652030701744546

3. Clark HA, Snedeker SM. Ochratoxin a: Its cancer risk and potential for exposure. J Toxicol Environ Health B Crit Rev 2006;9:265-96. http://dx.doi.org/10.1080/15287390500195570

4. Jennings-Gee JE, Tozlovanu M, Manderville R, et al. Ochratoxin A: in utero exposure in mice induces adducts in testicular DNA. Toxins (Basel) 2010;2:1428-44. http://dx.doi.org/10.3390/toxins2061428

5. Huff WE, Wyatt RD, Hamilton PB. Nephrotoxicity of dietary ochratoxin A in broiler chickens. Appl Microbiol 1975;30:48-51.

6. Patil RD, Dwivedi P, Sharma AK. Critical period and minimum single oral dose of ochratoxin A for inducing developmental toxicity in pregnant Wistar rats. Reprod Toxicol 2006;22:679-87. http://dx.doi. org/10.1016/i.reprotox.2006.04.022

7. Dwivedi P, Burns RB. Effect of ochratoxin A on immunoglobulins in broiler chicks. Res Vet Sci 1984:36:11721.

8. Mantle P, Kulinskaya E, Nestler S. Renal tumourigenesis in male rats in response to chronic dietary ochratoxin A. Food Addit Contam 2005;22(Suppl 1):58-64. http://dx.doi.org/10.1080/02652030500358431

9. Bendele AM, Carlton WW, Krogh P, et al. Ochratoxin A carcinogenesis in the (C57BL/6] X (3H)Fl mouse. J Natl Cancer Inst 1985;75:733-42.

10. Schwartz GG. Hypothesis: Does ochratoxin A cause testicular cancer? Cancer Causes Control 2002;13:91100. http://dx.doi.org/10.1023/A:1013973715289

11. IARC. Ochratoxin A. Some Naturally Occurring Substances: Food Items and Constituents, Heterocyclic Aromatic Amines and Mycotoxins IARC Monographs on the Evaluation of Carcinogenic Risk of Chemicals to Humans. Lyon, France: International Agency for Research on Cancer; 1993:489-521.
12. Kanisawa M. Synergistic effect of cltrinin on hepatorenal carcinogenesis of ochratoxin A in mice. Developments in Food Science 1984;7:245-54.

13. Krogh P. Mycotoxic porcine nephropathy: a possible model for Balkan endemic nephropathy. In: Puchlev A, editor. Endemic Nephropathy Proceedings of the second International Symposium on Endemic Nephropathy. Sofia: Bulgarian Academy of Science; 1974:266-70.

14. Petkova-Bocharova T, Castegnaro M. Ochratoxin A in human blood in relation to Balkan endemic nephropathy and urinary tract tumours in Bulgaria. IARC Sci Publ 1991:135-7.

15. Wafa EW, Yahya RS, Sobh MA, et al. Human ochratoxicosis and nephropathy in Egypt: a preliminary study. Hum Exp Toxicol 1998;17:124-9. http://dx.doi.org/10.1191/096032798678908369

16. Pfohl-Leszkowicz A, Petkova-Bocharova T, Chernozemsky IN, et al. Balkan endemic nephropathy and associated urinary tract tumours: a review on aetiological causes and the potential role of mycotoxins. Food Addit Contam 2002;19:282-302. http://dx.doi.org/10.1080/026520301 10079815

17. Abouzied MM, Horvath AD, Podlesny PM, et al. Ochratoxin A concentrations in food and feed from a region with Balkan Endemic Nephropathy. Food Addit Contam 2002;19:755-64. http://dx.doi. org/10.1080/02652030210145036

18. Hald B. Ochratoxin A in human blood in European countries. In: Castegnaro M, Plestina R, Dirheimer G, Chernozemsky IN, Bartsch H, editors. Mycotoxins, endemic nephropathy and urinary tract tumors. Lyon, France: International Agency for Research on Cancer; 1991:159-64.

19. Scott PM, Kanhere SR, Lau BP, et al. Survey of Canadian human blood plasma for ochratoxin A. Food Addit Contam 1998;15:555-62. http://dx.doi.org/10.1080/02652039809374681

20. Frohlich AA, Marquardt RR, Ominski KH. Ochratoxin A as a contaminant in the human food chain: a Canadian perspective. IARC Sci Publ 1991:139-43.

21. Grajewski J, Jarzemski P, Twaruzek M, et al. The level of ochratoxin a in patients after nephrectomy. Mycotoxin Research 2007;23:22-6. http://dx.doi.org/10.1007/BF02946020

22. Ozcelik N, Kosar A, Soysal D. Ochratoxin A in human serum samples collected in Isparta-Turkey from healthy individuals and individuals suffering from different urinary disorders. Toxicol Lett 2001;121:9-13. http://dx.doi.org/10.1016/S0378-4274(00)00291-5

23. Abid S, Hassen W, Achour A, et al. Ochratoxin A and human chronic nephropathy in Tunisia: is the situation endemic? Hum Exp Toxicol 2003;22:77-84. http://dx.doi.org/10.1191/0960327103ht3280a

24. Aslam M, Beg AE, Blaszkewicz $M$, et al. Ochratoxin A blood concentration in healthy subjects and bladder cancer cases from Pakistan. Mycotoxin Research 2005;21:164-7. http://dx.doi.org/10.1007/ BF02959255

25. Schwerdt G, Schuster C, Silbernag IS, et al. Preliminary results on Ochratoxin A concentrations in blood of patients with various kidney diseases in Germany. Mycotoxin Research 2001;17:146-9. http://dx.doi. org/10.1007/BF03036424

26. Pohland AE, Nesheim S, Friedman L. Ochratoxin A: A review (Technical report). Pure and Applied Chemistry 1992;64:1029-46. http://dx.doi.org/10.1351/pac199264071029

27. Mantle PG, Nolan CC. Pathological outcomes in kidney and brain in male Fischer rats given dietary ochratoxin a, commencing at one year of age. Toxins (Basel) 2010;2:1100-10. http://dx.doi.org/10.3390/ toxins 2051100

28. Boorman GA. Toxicology and Carcinogenesis Studies of Ochratoxin A (CAS No. 303-47-9) in F344/N Rats (Gavage Studies); National Toxicology Program Technical Report No. 358. NC, USA: National Institutes of Health; Research Triangle Park; 1989.

29. Studer-Rohr I, Schlatter J, Dietrich DR. Kinetic parameters and intraindividual fluctuations of ochratoxin A plasma levels in humans. Arch Toxicol 2000;74:499-510. http://dx.doi.org/10.1007/ s002040000157

30. Gazinska P, Herman D, Gillett C, et al. Comparative immunohistochemical analysis of ochratoxin A tumourigenesis in rats and urinary tract carcinoma in humans; mechanistic significance of $\mathrm{p}$-S6 ribosomal protein expression. Toxins (Basel) 2012;4:643-62. http://dx.doi.org/10.3390/toxins4090643

Correspondence: Dr. Stephen Pautler, Urology, St. Joseph's Hospital, 268 Grosvenor St., London, ON N6A 4V2; stephen.pautler@sihc.london.on.ca 